\title{
Searching for Axionlike Particles under Strong Gravitational Lenses
}

\author{
Aritra Basu๑, ${ }^{1,2, *}$ Jishnu Goswami ${ }^{2}$ Dominik J. Schwarz $\odot,{ }^{2}$ and Yuko Urakawa $\odot^{2,3}$ \\ ${ }^{1}$ Thüringer Landessternwarte, Sternwarte 5, 07778 Tautenburg, Germany \\ ${ }^{2}$ Fakultät für Physik, Universität Bielefeld, Postfach 100131, 33501 Bielefeld, Germany \\ ${ }^{3}$ Department of Physics and Astrophysics, Nagoya University, Chikusa, Nagoya 464-8602, Japan
}

(Received 10 July 2020; accepted 5 April 2021; published 11 May 2021)

\begin{abstract}
We establish strong gravitational lens systems as robust probes of axionlike particles (ALPs) —a candidate for dark matter. A tiny interaction of photons with ALPs induces birefringence. Multiple images of gravitationally lensed polarized objects allow measurement of differential birefringence, alleviating systematics and astrophysical dependencies. We apply this novel method to the lens system CLASS B1152 + 199 and constrain the ALP-photon coupling $\leq 9.2 \times 10^{-11}$ to $7.7 \times 10^{-8} \mathrm{GeV}^{-1}$ (95\% C.L.) for an ALP mass between $3.6 \times 10^{-21}$ and $4.6 \times 10^{-18} \mathrm{eV}$. A larger sample will improve the constraints.
\end{abstract}

DOI: $10.1103 /$ PhysRevLett.126.191102

Introduction.-The nature of dark matter remains elusive, as it interacts only weakly with visible matter. Various particles beyond the standard model have been proposed as dark matter candidates [1], wherein axions $[2,3]$ and axionlike particles (ALPs) are very promising [4-7]. The allowed mass range for ALPs spans tens of orders of magnitude, and massive efforts are underway to search for their signatures (see, e.g., [8-11]).

A promising direction of ALP searches focuses on its parity-violating interaction with photons through the coupling $g_{a \gamma}$, causing the left- and right-handed circular polarizations of light to propagate at different velocities in the ALP field-the birefringence phenomenon [12,13]. Consequently, the plane of polarization of linearly polarized light is rotated with respect to the plane at emission, and the amount of rotation $\Delta \theta_{a}$ depends on $g_{a \gamma}$. Since the ALP field oscillates in time with period $T_{a}=2 \pi / m_{a}$, where $m_{a}$ denotes the ALP mass, $\Delta \theta_{a}$ also oscillates and, thus, allows one to measure $m_{a}$.

ALP-induced birefringence is achromatic, and $\Delta \theta_{a}$ can be measured through observations of linearly polarizsed astrophysical systems. For example, linear polarization at $1.6 \mu \mathrm{m}$ wavelength caused by the scattering of light of the parent star in a protoplanetary disk [14], multiepoch observations at $2 \mathrm{~cm}$ of inherently linearly polarized synchrotron emission from knots in jets of active galactic nuclei (AGN) [15], and $E$-mode polarization of the cosmic microwave background radiation $(\mathrm{CMB})[11,16]$ have been used to constrain ultralight ALPs. Besides birefringence,

Published by the American Physical Society under the terms of the Creative Commons Attribution 4.0 International license. Further distribution of this work must maintain attribution to the author(s) and the published article's title, journal citation, and DOI. alternative approaches have also been used, such as interconversion between photons and ALPs in the presence of magnetic fields giving rise to modulations of $\mathrm{x}$-ray spectrum of AGN in a cluster [17,18] and probing gravitational effects of the oscillating ALP field by means of a pulsar timing array $[19,20]$.

In this Letter, we establish strong gravitational lens systems with polarized sources as a powerful new probe of ultralight ALPs via birefringence. Established astrophysical probes of ALPs heavily rely on assumptions and modeling or are limited by instrumental offset and sensitivity $[11,14,15]$ and are often sensitive to measure either $g_{a \gamma}[17]$ or $m_{a}$ [20]. Broadly speaking, they address different aspects -do ALPs exist and whether they are the dark matter. Here, we show that spectropolarimetric observations at gigahertz frequencies of strong gravitational lens systems, that produce multiple images of a polarized source, provide a unique advantage for probing ultralight ALP dark matter. Multiple images from lensing allow for performing differential polarization angle measurements, which alleviates instrumental offsets and does not rely on modeling the intrinsic astrophysics of the system. Differential measurements facilitate firm estimation of $m_{a}$ and $g_{a \gamma}$.

Differential measurements.-Measuring the birefringence angle is faced with two challenges-(i) accuracy of instrumental polarization angle calibration and (ii) additional chromatic birefringence introduced by Faraday rotation when linearly polarized light propagates through magnetized plasma. Although current instruments can measure the polarization angle to a fraction of a degree, the accuracy of absolute angle measurement is limited to a few degrees due to the accuracy to which the polarization angle of astronomical calibrators is known. The second challenge, Faraday rotation, depends on the photon frequency $\nu$, wherein the polarization angle is given as $\theta(\nu)=\theta_{0}+\operatorname{RM}(c / \nu)^{2} . \theta_{0}$ is the polarization angle of the 
source, and RM is the Faraday rotation measure. The effects of Faraday rotation are vastly reduced by performing observations at high frequencies ( $>100 \mathrm{GHz}$ ). However, at these frequencies very few astrophysical systems give rise to substantial linearly polarized emission.

In most astrophysical sources, linearly polarized emission at few-gigahertz frequencies directly originates from the synchrotron mechanism. However, in these frequencies, Faraday rotation introduces complicated frequency variation of the linear polarization parameters, Stokes $Q$ and $U$, when a polarized signal propagates through turbulent magnetized media [21]. These frequency variations are captured by polarization measurements performed over large bandwidths and are robustly modeled by applying the technique of Stokes $Q, U$ fitting. Stokes $Q, U$ fitting enables determination of the Faraday rotation-corrected polarization angle $\theta_{0}$ as one of the fitted parameters $[22,23]$. Since the birefringence induced by the interaction of photons with an ALP field is achromatic, the measured $\theta_{0}=\theta_{\mathrm{qso}}+\Delta \theta_{a}+\delta \theta_{\text {cal }} . \theta_{\mathrm{qso}}$ is the intrinsic polarization angle of a linearly polarized source, e.g., a quasar, and $\delta \theta_{\text {cal }}$ is the observational angle calibration offset. For observations of a quasar along a single line of sight, it is unfeasible to determine $\Delta \theta_{a}$ without the knowledge of $\theta_{\text {qso }}$ and $\delta \theta_{\text {cal }}$.

Strong gravitational lensing of polarized objects yields a unique advantage in mitigating the unknown $\theta_{\text {qso }}$ and $\delta \theta_{\text {cal }}$. Gravitational lensing offers the opportunity to simultaneously observe time-separated emission from a source, due to gravitational time delay, as lensed images. The timeseparated images encode the time variation of the oscillating ALP field at the emitting source, and, therefore, the polarization plane of each lensed image undergoes a different amount of birefringence, providing information on $\Delta \theta_{a}$. Polarized signals which were emitted at initial times $t_{i}$, with $i=A, B$ for the two images $A$ and $B$, are observed simultaneously at time $t_{\text {obs }}$. The gravitational time delay observed on Earth $\Delta t_{\mathrm{obs}}=\left|t_{A}-t_{B}\right|\left(1+z_{\mathrm{qso}}\right)$, where $z_{\mathrm{qso}}$ is the redshift of the lensed quasar. By performing Stokes $Q, U$ fitting separately for each image, the polarization angles of images $A$ and $B, \theta_{0, A}$ and $\theta_{0, B}$, respectively, can be obtained. Both $\theta_{0, A}$ and $\theta_{0, B}$ contain the same $\theta_{\text {qso }}$ and $\delta \theta_{\text {cal }}$ but different rotation angles, $\Delta \theta_{a, A}$ and $\Delta \theta_{a, B}$, arising from the time separation. Therefore, the differential angle $\Delta \theta_{a, \text { lens }}=\theta_{0, A}-\theta_{0, B}=\Delta \theta_{a, A}-\Delta \theta_{a, B}$ does not depend on $\theta_{\text {qso }}$ or $\delta \theta_{\text {cal }}$. The significance of the result is determined by statistical measurement noise. Birefringence in a similar setup was considered to explore the anomalous coefficient of axion strings [24] and for detecting cosmic axion background using polarized pulsars [25].

Differential birefringence.-We consider the Lagrangian density for an ALP field $a$ given as

$\mathcal{L}=-\frac{1}{4} F_{\mu \nu} F^{\mu \nu}-\frac{1}{2} \partial_{\mu} a \partial^{\mu} a+\frac{g_{a \gamma}}{4} a F_{\mu \nu} \tilde{F}^{\mu \nu}-\frac{1}{2} m_{a}^{2} a^{2}$, where $F_{\mu \nu}$ denotes the electromagnetic field strength tensor and $\tilde{F}^{\mu \nu}$ is its dual. We use the Heaviside-Lorentz system with $\hbar=c=1$. The equation of motion for $a$ is given by the Klein-Gordon equation, solved as

$$
a\left(t, x^{i}\right)=\frac{\sqrt{2 \rho_{a}\left(x^{i}\right)}}{m_{a}} \sin \left[m_{a} t+\delta\left(x^{i}\right)\right] .
$$

Here, $x^{i}$ represents the three spatial coordinates, $\rho_{a}$ is the energy density of the ALP field, and $\delta$ is the phase. Inhomogeneities of the ALP field are encoded in the spatial dependencies of $\rho_{a}$ and $\delta$, with $\delta$ being constant within patches of size of the de Broglie wavelength $\lambda_{\mathrm{dB}}$.

The parity-violating coupling term $a F \tilde{F}$ gives rise to birefringence. When temporal and spatial variations of the ALP field are much smaller than the frequency of the photons propagating in the ALP field, satisfying $10^{-16}\left(m_{a} / 10^{-22} \mathrm{eV}\right)(\mathrm{GHz} / \nu) \ll 1$, the rotation angle is given as $[11,12,16,26]$

$$
\Delta \theta_{a}=\frac{1}{2} g_{a \gamma}\left[a\left(t_{\mathrm{obs}}, x_{\mathrm{obs}}^{i}\right)-a\left(t_{\mathrm{em}}, x_{\mathrm{em}}^{i}\right)\right] .
$$

The subscripts "obs" and "em" indicate the ALP field at observation and at photon emission, respectively. Thus, in order to infer $g_{a \gamma}$, the field values at the emission region and at observations have to be known or assumed [15].

For $\Delta \theta_{a}$ measured toward two gravitationally lensed images $A$ and $B$, the field $a\left(t_{\mathrm{obs}}, x_{\mathrm{obs}}^{i}\right)$ is the same. In such a case, the differential birefringence angle $\Delta \theta_{a, \text { lens }}=\Delta \theta_{a, A}-$ $\Delta \theta_{a, B}$ depends only on the properties of ALP field in the emitting region and is not affected by the space-time curvature of the gravitational lens [26]. Thus, using Eqs. (2) and (3),

$$
\Delta \theta_{a, \text { lens }}=K \sin \left[\frac{m_{a} \Delta t}{2}\right] \sin \left(m_{a} t_{\mathrm{em}}+\delta_{\mathrm{em}}-\pi / 2\right) .
$$

Here, $K$ in normalized units is

$K=10^{\circ}\left[\frac{\rho_{a, \mathrm{em}}}{20 \mathrm{GeV} \mathrm{cm}^{-3}}\right]^{1 / 2} \frac{g_{a \gamma}}{10^{-12} \mathrm{GeV}^{-1}}\left[\frac{m_{a}}{10^{-22} \mathrm{eV}}\right]^{-1}$.

We have used $\rho_{a, A}=\rho_{a, B} \equiv \rho_{a, \mathrm{em}}$ (the ALP energy density in the emitting region), and $t_{\mathrm{em}}=\left(t_{A}+t_{B}\right) / 2$ is the mean time of emission. The phase difference $\delta_{\mathrm{em}}$ is the same for the emitting region. Unlike $\Delta \theta_{a}$, the amplitude of $\Delta \theta_{a \text {,lens }}$ depends on the lensing time delay in the frame of the emitting source, $\Delta t=\left|t_{A}-t_{B}\right|$. In Eq. (4), the $\sin \left(m_{a} t_{\mathrm{em}}+\delta_{\mathrm{em}}-\pi / 2\right)$ term suggests that $\Delta \theta_{a \text {, lens }}$ would also oscillate with the same time period as that of the ALP field determined by $m_{a}$ but shifted in phase by $90^{\circ}$. With sufficiently sensitive observations, the oscillation of 
$\Delta \theta_{a, \text { lens }}$ can be measured through monitoring of a lens system, and, thus, $m_{a}$ can be measured.

When $\Delta \theta_{a \text {, lens }}$ is measured at a single epoch, the ratio $g_{a \gamma} / m_{a}$ can be inferred for a given $\rho_{a}$ determined through ancillary measurements. This therefore provides $g_{a \gamma}$ over a range of $m_{a}$. The timescales involved in the emission, observations, and light propagation determine the range of ALP masses that can be probed. For a single epoch, $\Delta \theta_{a \text {,lens }}$ is given as $\Delta \theta_{a \text {, lens }}=K \sin \left(m_{a} \Delta t / 2\right) / \sqrt{2}$, where the factor $1 / \sqrt{2}$ originates from the root mean square of the oscillating ALP field with a random phase. Note that, in the case when $T_{a} \gg \Delta t$, the values of the field for images $A$ and $B$ would be close to each other and the differential birefringence would be negligible. Therefore, the lensing time delay determines the minimum ALP mass $\left(m_{a}=2 \pi / \Delta t\right)$ to which a single-epoch observation is sensitive. The maximum ALP mass is determined by the averaging time $t_{\text {avg }}$ of observations in the frame of the lensed object, as field oscillations on smaller timescales due to ALP with $m_{a}>$ $2 \pi / t_{\text {avg }}$ will average out in the observations.

When a lens system is regularly observed over a period of time $t_{\text {monitor }} \gg \Delta t$, the accessible mass range increases toward lower $m_{a}=2 \pi / t_{\text {monitor }}$. With sufficiently sensitive observations, the sinusoidal oscillation of $\Delta \theta_{a \text {, lens }}$ should be observed if $m_{a}$ is smaller than the corresponding time over which data are averaged in each of the observations. In the event of nondetection, limits obtained on $\Delta \theta_{a \text {, lens }}$ could extend the excluded parameter space compared to that obtainable for a single-epoch observation. A long monitoring program corresponds to the limit $m_{a} \Delta t \ll 1$, and, from Eq. (4), $\Delta \theta_{a \text {, lens }}$ can be constrained using the relation $\Delta \theta_{a \text {,lens }}=K m_{a} \Delta t / 2 \sqrt{2}$.

Constraint from CLASS B1152 + 199.-The lens system CLASS B1152 + 199 is a highly suitable candidate for probing ALPs and has been studied in detail in the literature. In the CLASS B1152 + 199 system, a foreground star-forming galaxy at redshift $z_{\text {gal }}=0.439$ lenses a background linearly polarized quasar at $z_{\mathrm{qso}}=1.019$ $[27,28]$. Using an isothermal sphere mass distribution model for the lensing galaxy, the best-fit time delay $\Delta t_{\mathrm{obs}}$ is estimated to be 27.8 days [28], which corresponds to $\Delta t=13.3$ days in the frame of the quasar [29].

Broadband polarization observations of this system, covering the frequency range $1-8 \mathrm{GHz}$, were performed using the Karl G. Jansky Very Large Array (VLA) to constrain the magnetic field geometry in the lensing galaxy [31]. The quasar is lensed into two images, denoted by image $A$ and $B$, separated by 1.56 arcsec. Both images were detected in the VLA observations, and their polarization spectra were independently fitted using the technique of Stokes $Q, U$ fitting [31]. It was found that the polarized emission of each of the images are composed of two polarization-emitting components which undergo Faraday rotation and depolarization in the magnetized plasma of the
TABLE I. Best-fit intrinsic polarization angle of the polarized emission components in CLASS B1152 + 199 taken from Table 1 of Ref. [31].

\begin{tabular}{lll}
\hline \hline Image & Component 1 & Component 2 \\
\hline$A$ & $\theta_{A, 1}=32^{\circ} \pm 1^{\circ}$ & $\theta_{A, 2}=-38^{\circ} \pm 4^{\circ}$ \\
$B$ & $\theta_{B, 1}=30^{\circ} \pm 10^{\circ}$ & $\theta_{B, 2}=-20^{\circ} \pm 20^{\circ}$ \\
\hline \hline
\end{tabular}

lensing galaxy. These polarized components, denoted as component 1 and 2, remained unresolved in the VLA observations at up to $0.5 \operatorname{arcsec}$ [31]. From milliarcsec resolution observations [28], these polarized components are physically separated by $<30 \mathrm{pc}$. The fitted angles of polarization of each of the polarized components in the lensed images are presented in Table I. We denote the Faraday rotation-corrected angles with the corresponding image and component as subscripts, i.e., $\theta_{I, C}$, where $I=A$, $B$ represents the lensed images and $C=1,2$ corresponds to the polarized components. Figure 1 illustrates the different polarization angles.

The presence of multiple polarized emission components in each of the lensed images allows us to measure three independent angle differences for the two lines of sight, i.e., two angle differences of the individual polarized components $\Delta \theta_{1}=\theta_{A, 1}-\theta_{B, 1}$ and $\Delta \theta_{2}=\theta_{A, 2}-\theta_{B, 2}$ and the difference of relative angles between the two polarized components $\Delta \theta_{12}=\left(\theta_{A, 1}-\theta_{A, 2}\right)-\left(\theta_{B, 1}-\theta_{B, 2}\right)$. Each of the angle differences measures $\Delta \theta_{a, \text { lens }}$, and the combined mean provides the net angle difference between the two lines of sight. This follows from the fact that emission from the components originating within $30 \mathrm{pc}$ is comparable to $\lambda_{\mathrm{dB}}$ for ALP dark matter with $m_{a} \sim 10^{-20} \mathrm{eV}$ and velocity dispersion $\sim 100 \mathrm{~km} \mathrm{~s}^{-1}$. The combined mean of the three
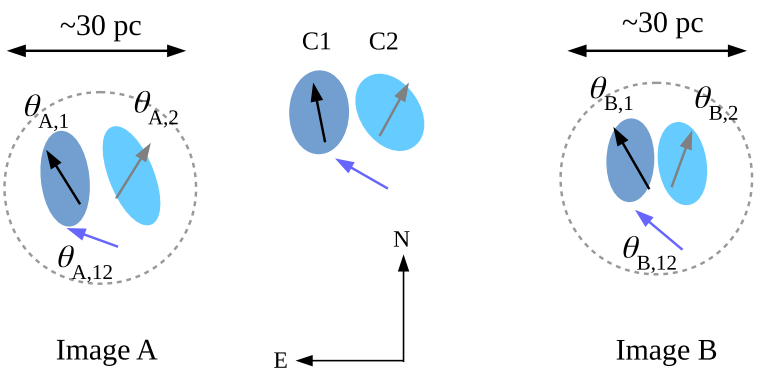

FIG. 1. Schematic of polarization angle orientations for the two lensed images of the background quasar in CLASS B1152 + 199. The angles $\theta_{A, i}$ and $\theta_{B, i}(i=1,2$, and 12) are used for our calculations. All angles are measured toward East from North of the coordinate axes shown here. The two polarized components are depicted as " $C 1$ " and " $C 2$." The dashed circles show a schematic spatial resolution of $\sim 30 \mathrm{pc}$ at the distance of the quasar. The relative shapes and orientations of the components in the lensed images are different to indicate shearing due to lensing. The extent of shearing of the components and their angles are exaggerated for representation and are not to scale. 
differential birefringence angles provides a net angle difference between the two lines of sight of $\Delta \theta_{a \text {, lens }}=$ $1.04_{-5.59}^{+7.67} \mathrm{deg}$ at $95 \%$ confidence level (C.L.), corresponding to the upper limit on the magnitude $\left|\Delta \theta_{a \text {, lens }}\right| \leq$ $8.71^{\circ}$ [32].

A part of $\Delta \theta_{a \text {, lens }}$ could arise due to the different light paths and differential gravitational shearing encountered by the two lensed images. Both effects are of the order of the lensing deflection angle [35]. For CLASS B1152 + 199, the deflection angle is 1.56 arcsec, significantly lower than the measured error for the angle difference between the two images. Hence, both effects are negligibly small. Furthermore, the total radio continuum intensity of CLASS B1152 + 199 at $8.46 \mathrm{GHz}$ was monitored using the VLA spanning over 7 months with an average observation spacing of 3.5 days [36]. No intensity variations of the two lensed images were observed. This strongly suggests that any changes in the polarization angles between the polarized components of the two images is unlikely to be due to intrinsic time variability of the lensed quasar.

Using the estimated limit on $\left|\Delta \theta_{a, \text { lens }}\right|, g_{a \gamma}$ can be constrained using Eq. (4). In Fig. 2, we present our constraint $g_{a \gamma} \leq 9.2 \times 10^{-11}\left(20 \mathrm{GeV} \mathrm{cm}^{-3} / \rho_{a, \mathrm{em}}\right)^{1 / 2} \mathrm{GeV}^{-1}$ to $7.7 \times$ $10^{-8}\left(20 \mathrm{GeV} \mathrm{cm}^{-3} / \rho_{a, \mathrm{em}}\right)^{1 / 2} \mathrm{GeV}^{-1}$ at $95 \%$ C.L. for the ALP mass range $3.6 \times 10^{-21} \mathrm{eV} \leq m_{a} \leq 4.6 \times 10^{-18} \mathrm{eV}$. As discussed earlier, the maximal ALP mass these

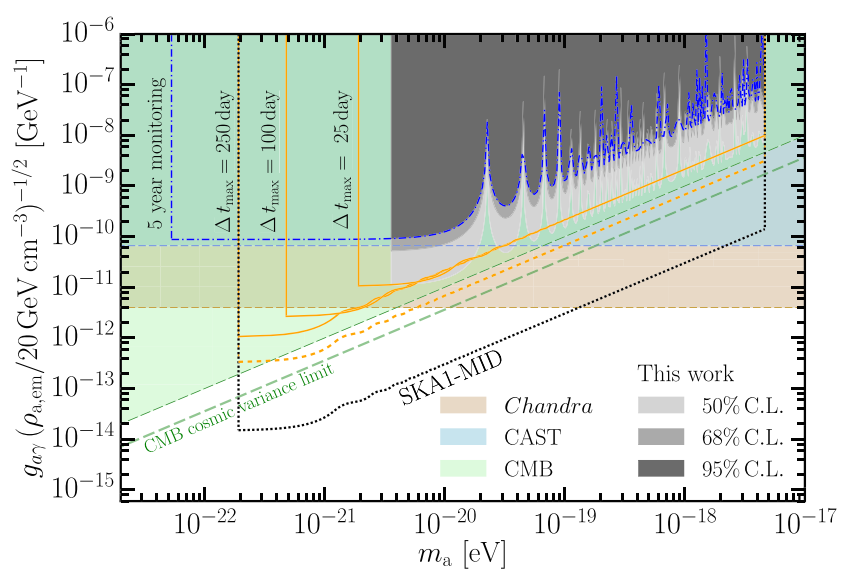

FIG. 2. Bounds on $g_{a \gamma}$ obtained from CLASS B $1152+199$ are shown as the shaded gray areas. The dash-dotted blue line shows the corresponding constraint that can be achieved if observations of CLASS B1152 + 199 are sampled over 5 yr. The solid and dashed orange lines show the constraint achievable for a sample of 100 and 1000 gravitational lens systems, respectively. The various $\Delta t_{\max }$ values for maximum time delay in the frame of the lensed source represent the respective constraints. The dotted black line shows the parameter space that can be probed by the SKA1-MID. For comparison, we show existing bounds at 95\% C.L. from the Chandra X-ray observations [17] (in brown), the CAST [37] (in blue), and CMB polarization [16] (in green) along with its cosmic variance limit. observations are sensitive to is determined by the observations averaging time of $30 \mathrm{~min}$ [31], i.e., $t_{\mathrm{avg}}=14.86 \mathrm{~min}$ corresponding to $m_{a}=4.6 \times 10^{-18} \mathrm{eV}$. The minimal $m_{a}$ probed is determined by the time delay $\Delta t$. For CLASS $\mathrm{B} 1152+199, \Delta t=13.3$ days corresponds to $m_{a}=$ $3.6 \times 10^{-21} \mathrm{eV}$.

Note that $\rho_{a \text { em }}$ is the only unknown quantity which can be assumed within an informed range when ALPs are the dark matter. Since quasars are hosted in elliptical galaxies, we infer $\left\langle\rho_{a, \text { em }}\right\rangle$ from the dark matter density measured in a sample of elliptical galaxies. The total matter density within the Einstein radii for a sample of elliptical galaxies has been measured through lensing and stellar kinematics [38,39]. Considering $50 \%$ of the mass in ellipticals is contributed by dark matter [40,41] and an ALP with $m_{a}>10^{-24} \mathrm{eV}$ comprises the entire dark matter [9], we estimate $\left\langle\rho_{a, \mathrm{em}}\right\rangle=$ $25 \mathrm{GeV} \mathrm{cm}^{-3}$ with dispersion $14.5 \mathrm{GeV} \mathrm{cm}^{-3}$. Consequently, we normalized $g_{a \gamma}$ using $\rho_{a, \mathrm{em}}=20 \mathrm{GeVcm}^{-3}$ in all the constraints presented in Fig. 2. A factor of 10 offset in the assumed $\rho_{a \text {,em }}$ would lead to systematic offset by a factor of $\sim 3$ in the values of $g_{a \gamma}$.

For single-epoch measurements, $m_{a}$ remains unknown, even if a detection of $\Delta \theta_{a \text {, lens }}$ would be obtained through sensitive follow-up observations of CLASS B1152 + 199 . Until such data are available, the blue dashed-dotted line in Fig. 2 shows the parameter space that can be probed by the CLASS B1152 + 199 system at 95\% C.L. if observations with similar sensitivity as used here are sampled over about $5 \mathrm{yr}$ (see above).

Statistical sample.-Constraints on $g_{a \gamma}$ probed using gravitational lensing can be vastly improved for a statistical sample. As this method makes minimal assumptions on astrophysical processes, the sample can consist of any type of lensing system where the lensed object is polarized. As the signal is measured via simple differences, $\Delta \theta_{a \text {,lens }}$ computed for individual lens systems can be simply combined to compute the mean differential birefringence angle $\left\langle\left|\Delta \theta_{a, \text { lens }}\right|\right\rangle$ of a sample of $N$ lens systems and reducing the uncertainty by $\mathcal{O}(1 / \sqrt{N})$.

In a sample of lensing systems, the time delay $\Delta t$ is a random variable, and $\left\langle\left|\Delta \theta_{a \text {, lens }}\right|\right\rangle$ is proportional to $\left\langle\left|\sin \left(m_{a} \Delta t / 2\right)\right|\right\rangle$, because the variables $\rho_{a, \text { em }}$ and $\Delta t$ are statistically independent. For a random argument of $\sin \left(m_{a} t_{\mathrm{em}}+\delta_{\mathrm{em}}-\pi / 2\right)$, the sample average $\left\langle\left|\sin \left(m_{a} t_{\mathrm{em}}+\delta_{\mathrm{em}}-\pi / 2\right)\right|\right\rangle=2 / \pi$. Assuming a uniform distribution for $\Delta t \in\left[0, \Delta t_{\max }\right],\left\langle\left|\Delta \theta_{a, \text { lens }}\right|\right\rangle$ is given by

$$
\begin{aligned}
\left\langle\left|\Delta \theta_{a, \text { lens }}\right|\right\rangle & =\frac{2 g_{a \gamma} \sqrt{2\left\langle\rho_{a, \mathrm{em}}\right\rangle}}{\pi m_{a} \Delta t_{\max }} \int_{0}^{\Delta t_{\max }}\left|\sin \left(\frac{m_{a} \Delta t}{2}\right)\right| d(\Delta t) \\
& =\frac{4 K}{\pi m_{a} \Delta t_{\max }}(2 n+1-\cos \zeta) .
\end{aligned}
$$

Here, $\Delta t_{\max }$ is the maximum time delay at the frame of lensed quasars in the sample of gravitational lens systems, $\left\langle\rho_{a, \mathrm{em}}\right\rangle$ is the sample mean energy density of the ALP field 
[42], and $K$ is the same as Eq. (5) except that $\rho_{a, \mathrm{em}}$ is replaced with $\left\langle\rho_{a \text {,em }}\right\rangle$. The angle $m_{a} \Delta t_{\max } / 2$ is expressed as $m_{a} \Delta t_{\max } / 2=n \pi+\zeta$, where $\zeta<\pi$ and $n \in \mathbb{Z}^{+}$because $|\sin x|$ has $n \pi$ periodicity.

The value of the sample mean birefringence angle depends on $\left\langle\rho_{a, \mathrm{em}}\right\rangle$, which can be inferred from the dark matter density in elliptical galaxies discussed earlier. Thus, the constraints on $g_{a \gamma}$ that can be obtained from a sample of gravitational lens systems suffer less systematics as compared to assuming a value of $\rho_{a, \text { em }}$ for a single lens system.

The solid (dashed) orange lines in Fig. 2 show the prediction for a sample of 100 (1000) gravitational lens systems having different values of $\Delta t_{\max }$ in the frame of the lensed object for $\left\langle\rho_{a \text {,em }}\right\rangle=20 \mathrm{GeV} \mathrm{cm}^{-3}$. Because of the large sample size, the sensitivity in measuring the birefringence angle improves by a factor of 10 (32) over the value obtained for CLASS B1152+199. The Square Kilometre Array-MID (SKA1-MID) is expected to detect about $10^{5}$ strong gravitational lens systems [43]. Assuming that 5\% of the systems will be polarized, we compute the parameter space that can be probed to constrain $g_{a \gamma}$ and is shown by the space above the dotted black line in Fig. 2. For all expected constraints, we have extrapolated the 95\% C.L. bound obtained for CLASS B1152 + 199. For the SKA1MID, we assume a 10 times better sensitivity compared to the VLA [44].

Future prospects.-For the ALP mass range probed in this Letter, the current strongest constraint on $g_{a \gamma}$ is obtained by investigating modulations of the x-ray spectrum of AGN in a cluster galaxy due to ALP-photon conversion induced by the cluster's magnetic fields [17] (brown region in Fig. 2). The limit $g_{a \gamma}<4 \times 10^{-12} \mathrm{GeV}^{-1}$ (95\% C.L.) depends on astrophysical parameters, such as structural properties of magnetic fields, free-electron density, and a model of the AGN's x-ray spectrum. The parameter space that can be probed by lensing using the SKA1-MID will improve upon existing bound on $g_{a \gamma}$ by almost 2 orders of magnitude for the mass range $10^{-22}$ to $10^{-20} \mathrm{eV}$ and by up to an order of magnitude in the mass range $10^{-20}$ to $10^{-19} \mathrm{eV}[11,16,17]$. This will complement the parameter space probed by the polarized CMB (green region in Fig. 2) and will even overcome cosmic variancelimited CMB constraints.

We have established differential birefringence from strong gravitational lensing as a new robust probe of ultralight ALPs which is sensitive to both $m_{a}$ and $g_{a \gamma}$. In contrast to the existing limit on $g_{a \gamma}$, constraints (and perhaps detection) of ALPs from differential birefringence measurements would be independent of astrophysical parameters and provide significantly stronger constraints compared to those provided by dedicated solar axion experiments, such as CAST [37] (blue region in Fig. 2). Future broad-bandwidth radio polarization observations of gravitational lensing systems using sensitive radio frequency observations will play a consequential role in our quest to understand the nature of dark matter.

We thank Pranjal Trivedi, Dietrich Bödeker, Giuseppe Gagliardi, Jordi Miralda Escudé, and Anirban Lahiri for insightful discussions on axion physics and ALP birefringence. We acknowledge financial support by the German Federal Ministry of Education and Research (BMBF) under Grant No. 05A17PB1 (Verbundprojekt D-MeerKAT) and from the Deutsche Forschungsgemeinschaft (DFG, German Research Foundation) through the CRC-TR 211 "Strong-interaction matter under extreme conditions" Project No. 315477589-TRR 211. Y. U. acknowledges support by JSPS KAKENHI Grants No. JP18H04349 and No. JP19H01894 and in part by No. YITP-T-19-02.

*abasu@tls-tautenburg.de

[1] G. Bertone and T. M. P. Tait, Nature (London) 562, 51 (2018).

[2] R. D. Peccei and H. R. Quinn, Phys. Rev. Lett. 38, 1440 (1977).

[3] F. Wilczek, Phys. Rev. Lett. 40, 279 (1978).

[4] J. E. Kim, Phys. Rev. Lett. 43, 103 (1979).

[5] M. Shifman, A. Vainshtein, and V. Zakharov, Nucl. Phys. B166, 493 (1980).

[6] M. Dine, W. Fischler, and M. Srednicki, Phys. Lett. 104B, 199 (1981).

[7] A. Zhitnitsky, Sov. J. Nucl. Phys. 31, 260 (1980), https:// inspirehep.net/literature/157263.

[8] P. W. Graham, I. G. Irastorza, S. K. Lamoreaux, A. Lindner, and K. A. van Bibber, Annu. Rev. Nucl. Part. Sci. 65, 485 (2015).

[9] R. Hlozek, D. Grin, D. J. E. Marsh, and P. G. Ferreira, Phys. Rev. D 91, 103512 (2015).

[10] I. G. Irastorza and J. Redondo, Prog. Part. Nucl. Phys. 102, 89 (2018).

[11] G. Sigl and P. Trivedi, arXiv:1811.07873.

[12] D. Harari and P. Sikivie, Phys. Lett. B 289, 67 (1992).

[13] S. M. Carroll, Phys. Rev. Lett. 81, 3067 (1998).

[14] T. Fujita, R. Tazaki, and K. Toma, Phys. Rev. Lett. 122, 191101 (2019).

[15] M. M. Ivanov, Y. Y. Kovalev, M. L. Lister, A. G. Panin, A. B. Pushkarev, T. Savolainen, and S. V. Troitsky, J. Cosmol. Astropart. Phys. 02 (2019) 059.

[16] M. A. Fedderke, P. W. Graham, and S. Rajendran, Phys. Rev. D 100, 015040 (2019).

[17] M. Berg, J. P. Conlon, F. Day, N. Jennings, S. Krippendorf, A. J. Powell, and M. Rummel, Astrophys. J. 847, 101 (2017).

[18] A. Ayad and G. Beck, J. Cosmol. Astropart. Phys. 04 (2020) 055 .

[19] A. Khmelnitsky and V. Rubakov, J. Cosmol. Astropart. Phys. 02 (2014) 019.

[20] N. K. Porayko, X. Zhu, Y. Levin, L. Hui, G. Hobbs et al., Phys. Rev. D 98, 102002 (2018).

[21] D. Sokoloff, A. Bykov, A. Shukurov, E. Berkhuijsen, R. Beck, and A. Poezd, Mon. Not. R. Astron. Soc. 299, 189 (1998). 
[22] S. O’Sullivan, S. Brown, T. Robishaw, D. Schnitzeler, N. McClure-Griffiths, I. Feain, A. Taylor, B. Gaensler, T. Landecker, L. Harvey-Smith, and E. Carretti, Mon. Not. R. Astron. Soc. 421, 3300 (2012).

[23] S. P. O’Sullivan, C. R. Purcell, C. S. Anderson, J. S. Farnes, X. H. Sun, and B. M. Gaensler, Mon. Not. R. Astron. Soc. 469, 4034 (2017).

[24] P. Agrawal, A. Hook, and J. Huang, J. High Energy Phys. 07 (2020) 138.

[25] T. Liu, G. Smoot, and Y. Zhao, Phys. Rev. D 101, 063012 (2020).

[26] D. J. Schwarz, J. Goswami, and A. Basu, Phys. Rev. D 103, 081306 (2021).

[27] S. T. Myers, D. Rusin, C. D. Fassnacht, R. D. Bland ford, T. J. Pearson, A. C. S. Readhead, N. Jackson, I. W. A. Browne, D. R. Marlow, P. N. Wilkinson, L. V. E. Koopmans, and A. G. de Bruyn, Astron. J. 117, 2565 (1999).

[28] D. Rusin, M. Norbury, A. D. Biggs, D. R. Marlow, N. J. Jackson, I. W. A. Browne, P. N. Wilkinson, and S. T. Myers, Mon. Not. R. Astron. Soc. 330, 205 (2002).

[29] Note that Ref. [28] computed $\Delta t_{\text {obs }}=39.7$ days for the assumed Hubble-Lemaitre constant $H_{0}=$ $100 \mathrm{~km} \mathrm{~s}^{-1} \mathrm{Mpc}^{-1}$. Here, we have corrected the estimated time delay using $H_{0}=67.4 \mathrm{~km} \mathrm{~s}^{-1} \mathrm{Mpc}^{-1}$ obtained from the Planck data [30] and used $\Delta t_{\mathrm{obs}}=27.8$ days in our calculations.

[30] Planck Collaboration VI, Astron. Astrophys. 641, A6 (2020).

[31] S. A. Mao, C. Carilli, B. M. Gaensler, O. Wucknitz, C. Keeton, A. Basu, R. Beck, P. P. Kronberg, and E. Zweibel, Nat. Astron. 1, 621 (2017).

[32] See Supplemental Material at http://link.aps.org/ supplemental/10.1103/PhysRevLett.126.191102 for details on estimation of the differential birefringence angle, which includes Refs. [33,34].
[33] N. Fisher, Statistical Analysis of Circular Data (Cambridge University Press, Cambridge, England, 1993).

[34] K. V. Mardia and P.E. Jupp, Directional Statistics (John Wiley \& Sons, Inc., New York, 2000).

[35] M. Lyutikov, Phys. Rev. D 95, 124003 (2017).

[36] N. Rumbaugh, C. D. Fassnacht, J. P. McKean, L. V. E. Koopmans, M. W. Auger, and S. H. Suyu, Mon. Not. R. Astron. Soc. 450, 1042 (2015).

[37] CAST Collaboration, Nat. Phys. 13, 584 (2017).

[38] L. V. E. Koopmans, T. Treu, A. S. Bolton, S. Burles, and L. A. Moustakas, Astrophys. J. 649, 599 (2006).

[39] N. Lyskova, E. Churazov, and T. Naab, Mon. Not. R. Astron. Soc. 475, 2403 (2018).

[40] N. Lyskova, E. Churazov, A. Moiseev, O. Sil'chenko, and I. Zhuravleva, Mon. Not. R. Astron. Soc. 441, 2013 (2014).

[41] M. R. Lovell, A. Pillepich, S. Genel, D. Nelson, V. Springel, R. Pakmor, F. Marinacci, R. Weinberger, P. Torrey, M. Vogelsberger, A. Alabi, and L. Hernquist, Mon. Not. R. Astron. Soc. 481, 1950 (2018).

[42] Here, we have made the approximation $\left\langle\rho_{a, \mathrm{em}}^{1 / 2}\right\rangle \approx\left\langle\rho_{a, \mathrm{em}}\right\rangle^{1 / 2}$. This is valid when $\left\langle\rho_{a, \mathrm{em}}\right\rangle$ is larger than the dispersion of $\rho_{a, \mathrm{em}}$.

[43] J. McKean, N. Jackson, S. Vegetti, M. Rybak, S. Serjeant, L. V. E. Koopmans, R. B. Metcalf, C. Fassnacht, P. J. Marshall, and M. Pandey-Pommier, in Advancing Astrophysics with the Square Kilometre Array (AASKA14) (Proceedings of Science, SISSA Medialabs, Trieste, 2015), p. 84, https://doi.org/10.22323/1.215.0084.

[44] R. Braun et al., SKA document number SKA-TELSKO-0000818, 2017, https://astronomers.skatelescope.org/ wp-content/uploads/2017/10/SKA-TEL-SKO-000081801\SKA1\Sciencel_Perform.pdf. 